\title{
5. SULFIDE MINERALOGY, SULFUR CONTENT, AND SULFUR ISOTOPE COMPOSITION OF MAFIC AND ULTRAMAFIC ROCKS FROM LEG $147^{1}$
}

\author{
H. Puchelt, ${ }^{2}$ H.M. Prichard, ${ }^{3}$ Z. Berner, ${ }^{2}$ and J. Maynard ${ }^{4}$
}

\begin{abstract}
A total of 107 hard-rock samples from Ocean Drilling Program Leg 147 drill cores were analyzed for sulfur content and, where possible, for sulfur isotope composition and sulfide mineralogy. At Site 894, plutonic rocks (gabbros and gabbronorites) from the roof of an intrarift magma chamber were sampled, whereas mafic and ultramafic rocks (gabbroic rocks, troctolites, dunites, and harzburgites) belonging to a transition zone between the lower magmatic crust and upper residual mantle were sampled at Site 895.

The average (and range) of sulfur content in analyzed rocks are as follows: gabbros (14 samples): 1590 ppm ( $<100-10340$ ppm); gabbronorites (39 samples): $760 \mathrm{ppm}(<100-3100 \mathrm{ppm}$ ); troctolites ( 9 samples): $680 \mathrm{ppm}(<100-1700 \mathrm{ppm}$ ); harzburgites (20 samples): $200 \mathrm{ppm}$ (<100-700 ppm); and dunites (19 samples): $400 \mathrm{ppm}(<100-1200 \mathrm{ppm})$.

About $65 \%$ of $\delta^{34} \mathrm{~S}$ values from 45 isotope analyses are between $-2 \%$ and $+4 \%$, which is similar to reported values of about $0 \% \circ \pm 2 \%$ for magmatic rocks and upper mantle material. Extreme deviations (up to $-29 \%$ ) from this range were found in three extremely serpentinized dunite samples.

In gabbroic rocks, sulfur-bearing phases were identified as pyrrhotite, pyrite (marcasite), and chalcopyrite by microscopy and electron microprobe. In troctolites, dunites dominate, and in harzburgites pentlandite dominates with some $\mathrm{Ni}-\mathrm{Fe}$ alloys and native copper as alteration products.
\end{abstract}

\section{INTRODUCTION}

Data on sulfur content and isotope composition of mantle rocks are scarce because few sampling locations are accessible on the surface. Ophiolites (Maynard, 1993) and xenoliths that are incorporated in volcanic materials during their ascent (Kovalenko et al., 1987; Chaussidon and Lorand, 1990; Lorand, 1991) have provided opportunities for these investigations to be conducted. Available data show that the sulfur content in mantle rocks is very low $(<50 \mathrm{ppm}$; Ionov et al., 1992). Sulfide minerals are rarely visible, normally with a size of only a few microns. The unique opportunity to sample a long sequence of mantle rocks is provided by two Ocean Drilling Program (ODP) Leg 147 cores, which probed $19.08 \mathrm{~m}$ of mantle at Hole 895D and $32.47 \mathrm{~m}$ at Hole $895 \mathrm{E}$.

Although data on the geochemistry of sulfur in upper parts of the oceanic crust are more readily available (e.g., Andrews, 1979; Hubberten and Puchelt, 1985; Alt et al., 1989), studies documenting the distribution and isotopic composition of sulfur in plutonic rocks of the lower oceanic crust are less abundant. Alt and Anderson (1991) focused on an area of slow spreading on the Southwest Indian Ridge (ODP Leg 118, Hole 735B). This hole consists of a 500-m-long profile through Layer 3, and is made up mainly of gabbros that show varying degrees of alteration. In contrast, beneath fast-spreading ridges, a different alteration regime was predicted in which high-temperature metamorphism may not play a significant role (Mével and

Mével, C., Gillis, K.M., Allan, J.F., and Meyer, P.S. (Eds.), 1996. Proc. ODP, Sci. Results, 147: College Station, TX (Ocean Drilling Program).

Institut für Petrographie und Geochemie der Universität Karlsruhe, Kaiserstrasse 12, D-7500 Karlsruhe, Federal Republic of Germany. dg09@rz.uni-karlsruhe.de

${ }^{3}$ Geology Department, University of Wales College of Cardiff, P.O. Box 914 Cardiff, CF1 3YE, Wales, United Kingdom. sglhmp@ cardiff.ac.uk

${ }^{4}$ Department of Earth Sciences, The Open University, Walton Hall, Milton Keynes, MK6 3AD, United Kingdom.
Cannat, 1991). At Site 894, located in an area of the fast-spreading Cocos-Nazca rift, rocks of the lower oceanic crust (mainly gabbroic rocks) were penetrated and sampled.

\section{GEOLOGIC SETTING AND LITHOSTRATIGRAPHY}

During Leg 147, two sites within the fast-spreading Cocos-Nazca rift valley at Hess Deep were investigated by drilling. An overview of the lithostratigraphy and geologic setting of the sampling sites is given by Mével, Gillis, and Shipboard Scientific Party (1993).

\section{Site 894}

Site 894 , located near the top of an intrarift ridge, revealed a sequence of gabbroic rocks, which is considered to have formed along the roof of an axial magma chamber. Of the seven holes drilled within an area of roughly 200 by $300 \mathrm{~m}$, Hole $894 \mathrm{G}$ provided by far the longest and most complete sequence of igneous rocks, penetrating to a depth of $154.5 \mathrm{~m}$ below seafloor (mbsf). Hole $894 \mathrm{~F}, 10 \mathrm{~m}$ to the east, represents a section of rock types equivalent to the upper $18.6 \mathrm{~m}$ of Hole 894G, which was drilled, but not cored in the latter hole. In the continuous section of the two holes, 13 lithologic units were recognized. The upper part of the profile is dominated by gabbros and olivine gabbros, deformed to various extents and locally transformed to cataclasites. Below $45 \mathrm{mbsf}$, gabbronorite is the principal lithology, interrupted only by a few short intervals of olivine gabbronorites and gabbros. Two porphyritic basaltic dikes were also recovered in Hole 894G.

The complex history of metamorphism and fluid-rock interaction at Site 894 starts with hydrothermal fluids of probable magmatic origin, resulting in the local development of coarse-grained amphibole pods. During the main stage of alteration, seawater-derived fluids penetrated along grain boundaries and microcracks generated by the thermal contraction of the cooling pluton. This pervasive static meta- 
morphism began with amphibolite facies and evolved to zeolite facies conditions. As a consequence, the primary mineral assemblage is at least $20 \%-50 \%$, but forms about $80 \%$ of the gabbroic rocks recovered in places totally replaced by newly formed mineral phases. Most characteristic for this background alteration stage is the transformation of pyroxenes. The fossilized channel ways of the altering fluids appear now as a ubiquitous network of microveins filled with amphibole. With progressive cooling, fluid circulation intensified, generating veins of macroscopic size with mineral assemblages formed at lower temperatures. In local cataclastic zones, Ca-rich fluids caused a pervasive alteration and recrystallization of the gabbroic host rocks from green schist to zeolite facies conditions.

\section{Site 895}

Site 895 lies about $9 \mathrm{~km}$ southeast of Site 894 on the southern slope of the same intrarift ridge. Only two of the six holes penetrated a depth greater than 40 mbsf (93.7 mbsf at Hole 895D and 87.6 mbsf at Hole $895 \mathrm{E}$ ). The sequence consists of an alternating series of peridotitic (dunites and harzburgites) and a lesser amount of mafic (mainly gabbroic) rocks and is considered to represent a transition zone between the lower magmatic crust and the upper residual mantle. In Hole $895 \mathrm{D}$, harzburgite is the main lithology to a depth of about 76 mbsf. Dunite together with more abundant sequences of gabbroic and troctolitic rocks occurs toward the bottom of the hole. Along the profile of Hole $895 \mathrm{E}$, dunite prevails over harzburgite (i.e., the relative proportion of peridotitic rocks is opposite that found in Hole 895D). Typically, a gradational transition between the two rock types could be observed. In dunites, short troctolitic sequences occur all along the transect, whereas gabbroic rocks (gabbros and olivine gabbros) are restricted mostly to its upper part. For the peridotitic rocks of Hess Deep there is a close association of dunite with refractory harzburgite. The harzburgites, as compared to other abyssal peridotites, are moderately to highly depleted.

The rocks of Site 895 are intensively altered by seawater-derived fluids. Static metamorphism evolved under somewhat lower thermal conditions as compared to those prevailing at Site 894 (greenschist to zeolitic facies). Alteration is strongly controlled by the primary mineralogy and in the peridotitic rocks is defined mainly by the replacement of olivine by serpentine. As a general rule, dunite is more intensively altered as compared to harzburgite. Several generations of veins composed mainly of serpentine and magnetite crosscut the pervasively altered background.

\section{SAMPLE PREPARATION AND ANALYTICAL TECHNIQUES}

Base-metal phases were described and tentatively identified using reflected light microscopy on the basis of their optical properties. Base-metal-bearing minerals from gabbros from Site 894 and troctolites, dunites, and harzburgites from Site 895 were analyzed using two techniques. Quantitative analyses of base-metal-rich phases were obtained using a Cambridge Mark 9 electron microprobe with a spot resolution size of approximately $5 \mu \mathrm{m}$. A subset of analyses was gained using a Cambridge 360 scanning electron microscope with a Link Systems energy-dispersive analytical attachment, specially calibrated for the quantitative analysis of sulfides with a spot resolution of approximately $2-3 \mu \mathrm{m}$. This subset of phases was analyzed because these phases are associated with platinum-group minerals.

Total sulfur content was determined on samples carefully washed with distilled water in order to remove seawater sulfate. Samples were ground to $<30 \mu \mathrm{m}$ in a shatter box with an agate lining and analyzed with a carbon-sulfur analyzer, type CSA-302 (Leybold Heraeus). About $200 \mathrm{mg}$ of sample were mixed with tungsten chips, which are used as accelerators. Sulfur in the sample material was converted to $\mathrm{SO}_{2}$ in a current of oxygen in a inductively heated furnace. The $\mathrm{SO}_{2}$ concentration was measured by infrared absorbency at a specific wavelength. The instrumental detection limit for sulfur is $50 \mathrm{ppm}$. Calibration was made against certified reference materials (Bundesanstalt für Materialprüfung, Berlin).

For $\mathrm{S}$ isotope determinations, a required minimum of $0.10 \mathrm{mmol}$ $\mathrm{S}$ must be converted to $\mathrm{SO}_{2}$. To obtain information about the isotopic signature of various sulfur-bearing minerals, they were first extracted chemically according to the scheme in Figure 1. Only the acid volatile sulfides (AVS: pyrrhotite, pentlandite, sphalerite, and, partially, chalcopyrite) and pyrite were separated in sufficient quantities for isotope analysis. $\mathrm{SO}_{2}$ was prepared according to Berner et al. (1993). Stochiometric mixtures of $\mathrm{CdS}$ and $\mathrm{V}_{2} \mathrm{O}_{5}$ were reacted at $1030^{\circ} \mathrm{C}$ in evacuated quartz tubes, and the evolved $\mathrm{SO}_{2}$ gas was analyzed for ${ }^{34} \mathrm{~S} /{ }^{\beta 2} \mathrm{~S}$ on a VG mass spectrometer with a dual inlet system (Micromass 1202). Results are reported relative to the CDT standard. The analytical uncertainty is typically less than $\pm 0.20 \%$.

\section{RESULTS Sulfide Mineralogy and Chemistry}

\section{Site 894}

In the gabbroic rocks of Site 894 , both primary as well as secondary sulfide phases occur. Primary sulfides are scarce and either form rounded, globular inclusions or are localized at the contact with interstitial oxide minerals. The larger proportion of the sulfides, disposed mostly in veins and patches after altered olivine and pyroxene, seems to have a secondary origin. Less commonly, these sulfides were found as inclusions in early crystallized minerals, in which case they replaced primary sulfides.

The most common base-metal sulfides in the gabbroic rocks are pyrrhotite, pyrite, marcasite, and chalcopyrite. Representative analyses are given from Samples 147-894G-7R-1 (Piece 11, 62-68 cm) and 147-894G-13R-2 (Piece 9A, 100-107 cm) (Table 1). Chalcopyrite (analysis 5, Table 1) commonly occurs within pyrrhotite (PI. 1, Fig. 1, and analysis 6, Table 1), which may be altered on the edges to pyrite (analysis 4 , Table 1 ). These sulfides are commonly associated with oxides (Pl. 1 and Fig. 2) and are typically interstitial to the host silicates. Quantitative analyses also confirmed the presence of much smaller, less than 5 - $\mu$ m-wide laths of pentlandite in the alteration zones adjacent to olivine in olivine gabbros.

In sections from Site $894 \mathrm{G}$ where gabbro is adjacent to basaltic dikes, the greater degree of alteration suggests a higher water to rock ratio. Core locations where this greater alteration occurs are Sections 147-894G-2R-1, 147-894G-2R-2, and 147-894G-19R-1. Euhedral zoned sphalerite was observed in prehnite veins crosscutting the gabbro (Sample 147-894G-2R-3 [Piece 3, 11-14 cm]). In these zones, pyrite is commonly altered to marcasite, which has a characteristic mottled extinction and usually has an irregular outline.

In the basaltic dikes, pyrite and to a lesser amount chalcopyrite are the most representative sulfides and they commonly occur in the more altered zones.

\section{Site 895}

Sulfide minerals in the ultramafic and gabbroic lithologies of Site 895 occur as veins or as secondary phases associated with alteration products after clinopyroxene. Pentlandite and pyrite are the dominant sulfides in these rocks.

\section{Troctolites}

The most common base-metal sulfide in the troctolites is pentlandite, which occurs as grains within spinels (PI. 1, Fig. 4), interstitial subangular grains between olivine crystals (PI. 1, Fig. 5), and much 


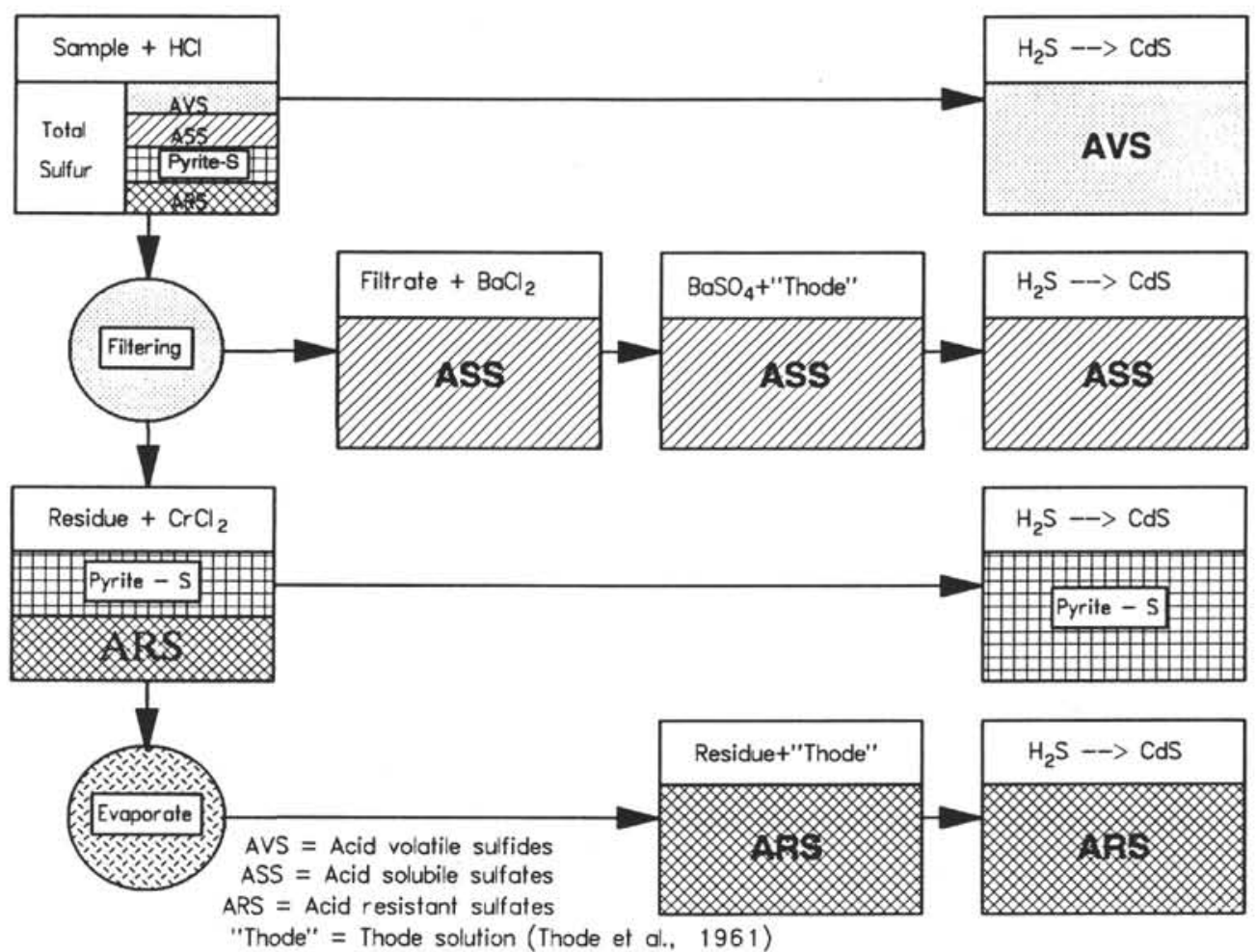

Figure 1. Chemical preparation scheme used to separate sulfur-bearing phases for isotopic analyses.

more rarely within altered plagioclase. The pentlandite is commonly partially altered to awaruite, a Ni-Fe alloy or millerite. Chalcopyrite is associated with the pentlandite but is usually replaced by native copper, which is characteristically situated around the $\mathrm{Ni}$-Fe-bearing minerals. For example, in Sample 147-895C-4R-2 (Piece 6C, 129$133 \mathrm{~cm}$ ), native copper is adjacent to pentlandite. In analysis 15 (Table 1), the native copper appears to contain minor $\mathrm{Ni}, \mathrm{Fe}$, and $\mathrm{S}$, which may be contributed to the analysis from the surrounding $\mathrm{Ni}$ Fe-bearing minerals.

\section{Dunites}

The most abundant sulfide in dunite is also pentlandite (analyses $22-25$, Table 1$)$ which is commonly partially altered to awaruite $(\mathrm{Pl}$. 1, Fig. 6, and analyses 27 and 28, Table 1).

\section{Harzburgites}

The harzburgites contain diverse base-metal-bearing minerals as varied as in the other lithologies. Again, the most common mineral is pentlandite, which usually contains laths of magnetite and is commonly altered to awaruite (analyses 16 and 17, Table 1). In one case, a cobaltian pentlandite was analyzed (analysis 26 , Table 1). Some chalcopyrite is altered to bornite and native copper. Rare marcasite is also present in the serpentinites at Site 895 (Pl. 1, Fig. 3).

\section{Sulfur Contents}

The sulfur contents and isotope data of the analyzed samples are listed in Table 2. Mean values and variation limits grouped according to lithology are summarized in Table 3.

\section{Site 894}

Of all the samples analyzed, the gabbros and gabbronorites at Site 894 display the highest sulfur contents (Fig. 2). The average sulfur concentration in the gabbronorites (i.e., in the main lithology of Hole
$894 \mathrm{G}$ ) is $760 \mathrm{ppm}$ ( 39 samples). Two samples lie below the detection limit (100 ppm), and at the higher end, only one surpasses $2000 \mathrm{ppm}$ (3160 ppm). Concentrations in the gabbros are quite similar. If two olivine gabbro samples that are extremely enriched in sulfur are not considered, an almost identical mean value of $780 \mathrm{ppm}$ results. These averages are only slightly higher than those found by Alt and Anderson (1991) in Layer 3 gabbros from Hole 735B (Southwestern Indian Ocean). Extreme $\mathrm{S}$ contents are commonly associated with zones of intensive alteration. In Samples 147-894G-2R-3 (Piece 1, 0-5 cm) and (Piece 4, 20-26 cm) with the highest sulfur contents (4570 and $10,340 \mathrm{ppm})$, the modal alteration attains its highest value $(84.8 \%)$. Here, in a cataclastic shear zone developed in highly altered gabbros, pyrite-sphalerite-chalcopyrite are associated with composite chlorite-prehnite veins.

\section{Site 895}

In contrast, the suite of peridotitic rocks at Site 895 is significantly lower in sulfur. The average S content in harzburgite, dunite, and troctolite was found to be 200,400 , and $680 \mathrm{ppm}$, respectively, but strongly serpentinized samples are distinctly higher (Table 3 ). Less altered harzburgites and dunites show average concentrations (100 and $230 \mathrm{ppm}$, respectively) that are close to the upper mantle estimates of 200-350 ppm based on partial-melting model calculations (Sun, 1982; Ringwood and Kesson, 1976) and studies on orogenictype peridotites (Garuti et al., 1984; Lorand, 1989, 1991; Chaussidon and Lorand, 1990). At the same time, they significantly exceed the values (commonly $<50-60 \mathrm{ppm}$ ) of peridotitic xenoliths (Mitchell and Keays, 1981; Lorand, 1990; Hartmann and Wedepohl, 1990; Ionov et al., 1992). The troctolites are always almost completely transformed into an association of secondary minerals and have sulfur concentrations that are unusually high for this sequence of rocks. For example, Sample 147-895E-4R-1 (Piece 7, 34-36 cm), which has the highest $\mathrm{S}$ content $(1750 \mathrm{ppm})$, is from a completely altered troctolite in which secondary sulfides are more abundant than anywhere else in 
Table 1. Quantitative electron microprobe analyses of base-metal-rich phases.

\begin{tabular}{|c|c|c|c|c|c|c|c|c|c|c|}
\hline $\begin{array}{l}\text { Analysis } \\
\text { number }\end{array}$ & $\begin{array}{l}\text { Core, section, } \\
\text { interval }(\mathrm{cm})\end{array}$ & Piece & $\mathrm{Fe}$ & $\mathrm{Ni}$ & Co & $\mathrm{S}$ & $\mathrm{Cu}$ & Total & Mineral & Lithology \\
\hline & $147-894 \mathrm{G}-$ & & & & & & & & & \\
\hline 1 & 7R-1, 62-68 & 11 & 30.4 & 0.0 & 0.1 & 33.9 & 33.9 & 98.3 & Chalcopyrite & Olivine gabbro \\
\hline 2 & $7 R-1,62-68$ & II & 57.1 & 2.1 & 0.3 & 38.1 & 0.0 & 97.6 & Pyrrhotite & Olivine gabbro \\
\hline 3 & $7 R-1,62-68$ & 11 & 56.8 & 2.3 & 0.3 & 39.1 & 0.1 & 98.6 & Pyrrhotite & Olivine gabbro \\
\hline 4 & $13 R-2,100-107$ & $9 \mathrm{~A}$ & 30.3 & 0.0 & 0.0 & 34.5 & 34.6 & 99.4 & Chalcopyrite & Gabbro \\
\hline 5 & $13 \mathrm{R}-2,100-107$ & $9 \mathrm{~A}$ & 30.0 & 0.3 & 0.1 & 34.6 & 34.5 & 99.5 & Chalcopyrite & Gabbro \\
\hline 6 & $13 \mathrm{R}-2,100-107$ & $9 \mathrm{~A}$ & 40.9 & 3.4 & 0.1 & 41.0 & 0.2 & 85.6 & Pyrrhotite & Gabbro \\
\hline 7 & $13 \mathrm{R}-2,100-107$ & $9 \mathrm{~A}$ & 45.7 & 0.0 & 1.3 & 53.9 & 0.1 & 101.0 & Pyrite & Gabbro \\
\hline 8 & $13 R-2,100-107$ & $9 \mathrm{~A}$ & 46.5 & 0.0 & 0.1 & 53.5 & 0.1 & 100.2 & Pyrite & Gabbro \\
\hline 9 & $13 R-2,100-107$ & $9 \mathrm{~A}$ & 42.1 & 5.4 & 0.5 & 53.7 & 0.1 & 101.8 & Pyrite & Gabbro \\
\hline 10 & $13 \mathrm{R}-2,100-107$ & $9 \mathrm{~A}$ & 45.1 & 0.0 & 1.8 & 52.7 & 0.0 & 99.6 & Pyrite & Gabbro \\
\hline \multirow[t]{2}{*}{11} & $13 \mathrm{R}-2,100-107$ & $9 \mathrm{~A}$ & 45.9 & 0.9 & 0.1 & 49.2 & 0.0 & 96.1 & Pyrite & Gabbro \\
\hline & 147-895C- & & & & & & & & & \\
\hline 12 & $4 \mathrm{R}-2,129-133$ & $6 \mathrm{C}$ & 31.9 & 33.1 & 1.3 & 31.5 & 0.0 & 97.8 & Pentlandite & Troctolite \\
\hline 13 & $4 \mathrm{R}-2,129-133$ & $6 \mathrm{C}$ & 35.9 & 28.1 & 1.3 & 32.5 & 1.1 & 98.9 & Pentlandite & Troctolite \\
\hline 14 & $4 \mathrm{R}-2,129-133$ & $6 \mathrm{C}$ & 22.5 & 72.8 & 0.3 & 0.0 & 2.6 & 98.2 & Awaruite & Troctolite \\
\hline \multirow[t]{2}{*}{15} & $4 \mathrm{R}-2,129-133$ & $6 \mathrm{C}$ & 6.7 & 5.6 & 0.1 & 6.6 & 81.7 & 100.7 & Native copper & Troctolite \\
\hline & 147-895D- & & & & & & & & & \\
\hline 16 & $3 \mathrm{R}-1,133-139$ & 15 & 28.9 & 50.9 & 0.2 & 17.4 & 0.1 & 97.5 & Pentlandite/awaruite & Harzburgite \\
\hline 17 & $3 \mathrm{R}-1,133-139$ & 15 & 27.1 & 48.2 & 0.2 & 20.8 & 0.1 & 96.4 & Pentlandite/awaruite & Harzburgite \\
\hline 18 & $7 \mathrm{R}-1,71-74$ & 11 & 35.3 & 28.3 & 0.8 & 32.2 & 3.0 & 99.6 & Pentlandite & Troctolite \\
\hline 19 & $7 \mathrm{R}-1.71-74$ & 11 & 34.1 & 30.2 & 0.9 & 29.2 & 6.3 & 100.7 & Pentlandite & Troctolite \\
\hline 20 & $7 \mathrm{R}-1,71-74$ & 11 & 36.1 & 28.8 & 0.7 & 32.6 & 1.7 & 99.9 & Pentlandite & Troctolite \\
\hline 21 & $7 R-2,8-12$ & 2 & 10.6 & 65.5 & 0.8 & 27.7 & 0.1 & 104.7 & Pentlandite/awaruite & Harzburgite \\
\hline 22 & $10 \mathrm{~W}, 25-28$ & 3 & 33.3 & 31.8 & 1.3 & 32.1 & 0.0 & 98.5 & Pentlandite & Dunite \\
\hline 23 & $10 \mathrm{~W}, 25-28$ & 3 & 33.0 & 32.2 & 1.5 & 32.2 & 0.0 & 98.9 & Pentlandite & Dunite \\
\hline 24 & $10 \mathrm{~W}, 25-28$ & 3 & 32.3 & 33.0 & 1.3 & 31.6 & 0.0 & 98.2 & Pentlandite & Dunite \\
\hline \multirow[t]{2}{*}{25} & $10 \mathrm{~W}, 25-28$ & 3 & 32.4 & 33.2 & 1.4 & 32.8 & 0.0 & 99.8 & Pentlandite & Dunite \\
\hline & 147-895E- & & & & & & & & & \\
\hline 26 & IR-3, 74-76 & 3B & 13.6 & 25.9 & 25.7 & 30.8 & 0.0 & 96.0 & Co-pentlandite & Harzburgite \\
\hline 27 & $4 \mathrm{R}-2,105-109$ & 12 & 27.1 & 73.5 & 0.7 & 0.0 & 0.3 & 101.6 & Awaruite & Dunite \\
\hline 28 & $4 \mathrm{R}-2,105-109$ & 12 & 25.3 & 73.4 & 0.6 & 0.0 & 0.3 & 99.6 & Awaruite & Dunite \\
\hline 29 & $6 \mathrm{R}-1,90-92$ & 17 & 29.0 & 37.1 & 0.0 & 31.6 & 0.4 & 98.1 & Pentlandite & Troctolite \\
\hline 30 & $6 \mathrm{R}-1,90-92$ & 17 & 23.9 & 72.6 & 0.0 & 0.2 & 1.2 & 97.9 & Awaruite & Troctolite \\
\hline 31 & $6 R-1,90-92$ & 17 & 24.2 & 73.6 & 0.0 & 0.0 & 1.2 & 99.0 & Awaruite & Troctolite \\
\hline 32 & $6 \mathrm{R}-1,90-92$ & 17 & 3.1 & 68.9 & 0.0 & 25.4 & 0.0 & 97.4 & Millerite & Troctolite \\
\hline 33 & $6 \mathrm{R}-1,90-92$ & 19 & 25.6 & 73.8 & 0.0 & 0.0 & 0.6 & 100.0 & Awaruite & Dunite \\
\hline
\end{tabular}

Note: Because the base-metal-rich phases in Samples 147-895E-6R-1, 90-92 cm, and 147-895E-4R-2, 105-109 cm, are associated with platinum-group minerals, these analyses were obtained using a scanning electron microscope equipped with an energy-dispersive analytical attachment.

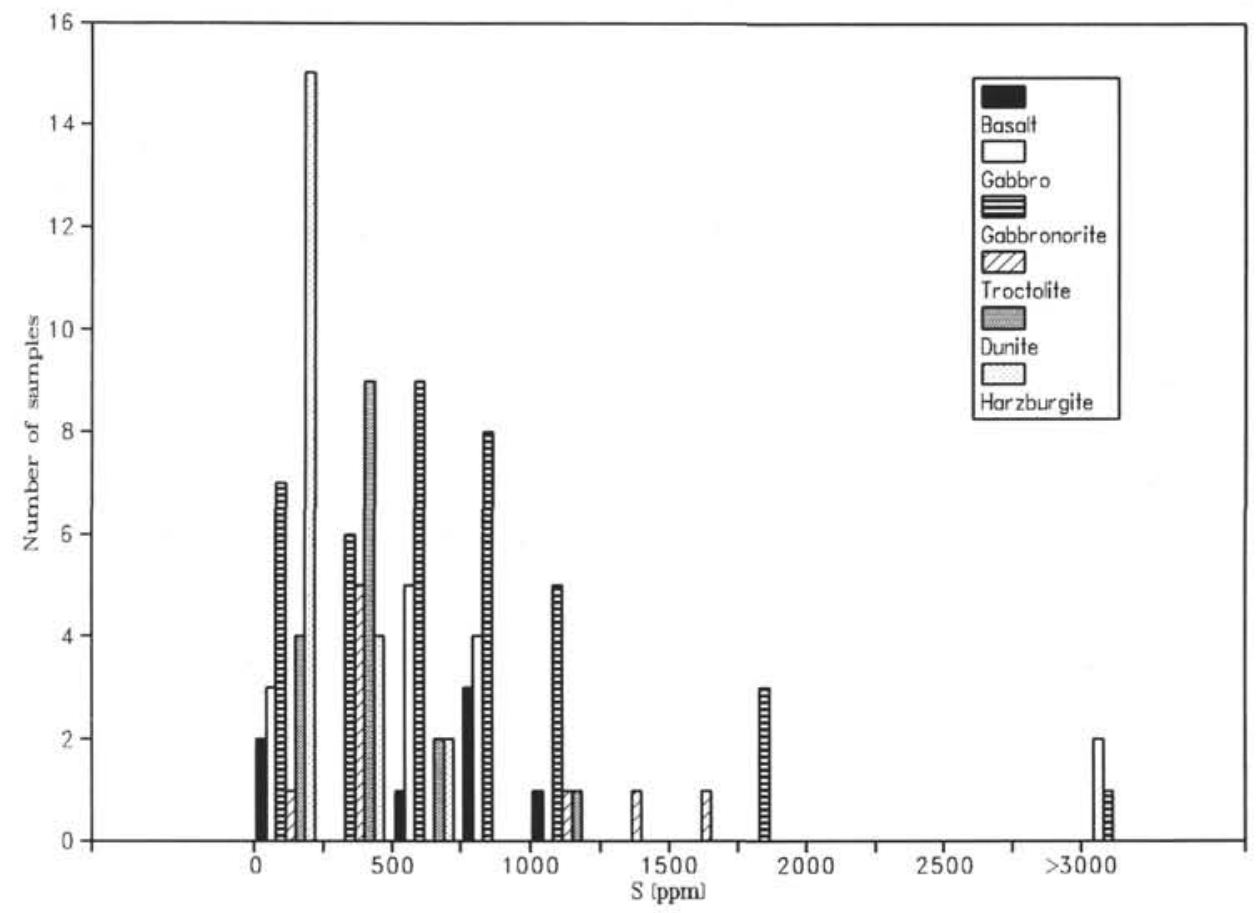

Figure 2. Histogram showing the distribution of sulfur contents in various lithologies. 
Table 2. Sulfur content and $\mathrm{S}$ isotope values in samples from Sites 894 and 895.

\begin{tabular}{|c|c|c|c|c|c|c|c|}
\hline \multirow[b]{2}{*}{$\begin{array}{l}\text { Core, section, } \\
\text { interval }(\mathrm{cm})\end{array}$} & \multirow[b]{2}{*}{$\begin{array}{l}\text { Depth } \\
\text { (mbsf) }\end{array}$} & \multirow[b]{2}{*}{ Lithology } & & $\%$ of $S$ & ixed as & $\delta^{34} s$ & $8^{34} \mathrm{~S}$ \\
\hline & & & $\begin{array}{c}\text { content } \\
\text { (ppm) }\end{array}$ & AVS & Pyrite & AVS & Pyrite \\
\hline $147-894 \mathrm{E}-$ & & & & & & & \\
\hline $3 R-1,19-21$ & 19.09 & Gabbro & 700 & & & +3.8 & -8.6 \\
\hline $3 \mathrm{R}-1,21-25$ & 19.11 & Gabbro & 960 & & & & \\
\hline $3 R-1,62-70$ & 19.52 & Gabbro & 550 & & & & \\
\hline $147-894 \mathrm{~F}-$ & & & & & & & \\
\hline $3 R-1,98-103$ & 17.79 & Gabbro & 590 & & & & \\
\hline $3 \mathrm{R}-1,99-101$ & 17.79 & Gabbro & 1000 & & & & \\
\hline $147-894 \mathrm{G}-$ & & & & & & & \\
\hline $2 \mathrm{R}-1,8-10$ & 28.68 & Olivine gabbro & 900 & 99.3 & 0.7 & +0.5 & - \\
\hline $2 \mathrm{R}-1,60-65$ & 29.20 & Basalt & 760 & & & & \\
\hline $2 \mathrm{R}-1,127-134$ & 29.87 & Basalt & 780 & 83.0 & 17.0 & -1.1 & $(+1.0)$ \\
\hline $2 \mathrm{R}-2,95-100$ & 31.05 & Basalt & 800 & & & & \\
\hline $2 \mathrm{R}-2,113-115$ & 31.23 & Basalt & 700 & & & & \\
\hline $2 \mathrm{R}-3,0-5$ & 31.55 & Olivine gabbro & 4570 & & & & \\
\hline $2 \mathrm{R}-3,20-26$ & 31.75 & Olivine gabbro & 10340 & 24.1 & 75.9 & - & +4.2 \\
\hline $2 \mathrm{R}-3,109-115$ & 32.64 & Olivine gabbro & 750 & & & & \\
\hline $2 \mathrm{R}-3,120-122$ & 32.75 & Olivine gabbro & 900 & & & & \\
\hline $4 \mathrm{R}-1,113-119$ & 46.15 & Gabbronorite & 1080 & & & & \\
\hline $5 R-1,10-14$ & 50.10 & Gabbronorite & 400 & & & & \\
\hline $5 \mathrm{R}-1,27-30$ & 50.27 & Gabbronorite & 1100 & 25.9 & 74.1 & -2.0 & -0.7 \\
\hline $5 R-1,30-34$ & 50.30 & Gabbronorite & 600 & & & & \\
\hline $6 \mathrm{R}-1,81-85$ & 55.61 & Gabbronorite & 600 & 1.8 & 98.2 & - & +4.4 \\
\hline $6 \mathrm{R}-2,12-14$ & 56.35 & Gabbronorite & 3100 & 4.2 & 95.8 & -9.0 & +2.0 \\
\hline $6 R-2,79-87$ & 57.02 & Gabbronorite & 620 & 82.5 & 17.5 & +1.5 & +3.0 \\
\hline $6 \mathrm{R}-2,113-117$ & 57.36 & Gabbronorite & 1800 & & & & \\
\hline $6 \mathrm{R}-2,141-143$ & 57.64 & Gabbronorite & 1900 & & & & \\
\hline $7 R-1,56-66$ & 65.36 & Gabbronorite & 990 & 44.9 & 55.1 & +2.1 & +1.7 \\
\hline $7 \mathrm{R}-1,75-76$ & 65.55 & Gabbronorite & 100 & & & & \\
\hline $7 \mathrm{R}-1,76-84$ & 65.56 & Gabbronorite & 130 & & & & \\
\hline $9 R-2,28-29$ & 75.93 & Gabbronorite & 300 & 79.1 & 20.9 & +5.9 & +4.0 \\
\hline $9 R-2,115-120$ & 76.80 & Gabbronorite & $<100$ & & & & \\
\hline $9 \mathrm{R}-3,133-139$ & 78.32 & Gabbronorite & 780 & & & & \\
\hline $9 \mathrm{R}-4,100-144$ & 79.50 & Olivine gabbronorite & 710 & 97.7 & 2.3 & -0.5 & - \\
\hline $9 R-4,127-129$ & 79.60 & Olivine gabbronorite & 1000 & & & & \\
\hline 10R-1, 90-91 & 80.00 & Gabbro & 700 & & & & \\
\hline $11 \mathrm{R}-2,100-107$ & 86.60 & Gabbronorite & 200 & & & & \\
\hline $11 \mathrm{R}-2,133-137$ & 86.89 & Gabbronorite & 1040 & & & & \\
\hline IIR-2, 138-140 & 86.94 & Gabbronorite & 900 & 53.6 & 46.4 & -0.3 & -0.7 \\
\hline $11 \mathrm{R}-3,17-28$ & 87.14 & Gabbronorite & 1040 & & & & \\
\hline $11 \mathrm{R}-3,31-38$ & 87.28 & Gabbronorite & 660 & 15.2 & 84.8 & $(+3.7)$ & +2.1 \\
\hline $12 \mathrm{R}-2,37-38$ & 95.63 & Gabbronorite & 200 & & & & \\
\hline $12 \mathrm{R}-2,37-45$ & 95.83 & Gabbronorite & 800 & 4.3 & 95.7 & - & +3.5 \\
\hline $12 \mathrm{R}-3,40-45$ & 97.13 & Gabbronorite & 600 & 1.4 & 98.6 & - & +2.0 \\
\hline 12R-5, 84-88 & 100.57 & Gabbronorite & 800 & & & & \\
\hline $13 \mathrm{R}-1,42-48$ & 103.85 & Gabbronorite & 150 & & & & \\
\hline $13 \mathrm{R}-2,6-13$ & 104.83 & Gabbronorite & $<100$ & & & & \\
\hline $13 \mathrm{R}-2,90-98$ & 105.67 & Gabbronorite & 340 & 22.0 & 78.0 & - & $(+4.1)$ \\
\hline $13 \mathrm{R}-2,100-107$ & 105.77 & Gabbronorite & 980 & 8.7 & 91.3 & $(+1.0)$ & +1.1 \\
\hline $13 \mathrm{R}-2,132-137$ & 106.09 & Gabbronorite & 300 & & & & \\
\hline $13 \mathrm{R}-3,13-15$ & 106.36 & Gabbronorite & 300 & 4.3 & 95.7 & - & +1.3 \\
\hline $13 R-3,17-24$ & 106.40 & Gabbronorite & 530 & & & & \\
\hline $13 \mathrm{R}-3,131-134$ & 107.54 & Gabbronorite & 500 & 2.1 & 97.9 & - & +2.6 \\
\hline $17 \mathrm{R}-1,50-58$ & 126.30 & Gabbronorite & 640 & 22.8 & 77.2 & $(+3.5)$ & +2.0 \\
\hline 17R-1, 95-99 & 126.75 & Gabbronorite & 800 & & & & \\
\hline $19 \mathrm{R}-1,49-55$ & 141.04 & Gabbronorite & 1860 & 1.7 & 98.3 & - & +4.4 \\
\hline $19 \mathrm{R}-1,84-96$ & 141.39 & Gabbronorite & 530 & 23.6 & 76.4 & +9.2 & +5.6 \\
\hline $20 \mathrm{R}-1,19-26$ & 145.83 & Gabbronorite & 1170 & & & & \\
\hline $147-895 \mathrm{~A}-$ & & & & & & & \\
\hline $2 \mathrm{R}-1,19-26$ & 9.36 & Basalt & 200 & & & & \\
\hline 2R-1,91-95 & 10.08 & Basalt & 1020 & & & & \\
\hline 147-895B- & & & & & & & \\
\hline IR-1, 77-81 & 0.77 & Harzburgite (serp.) & 200 & & & - & - \\
\hline $147-895 \mathrm{C}$ - & & & & & & & \\
\hline $2 \mathrm{R}-1,2-4$ & 12.32 & Harzburgite & 100 & & & & \\
\hline $4 \mathrm{R}-1,129-133$ & 29.95 & Harzburgite & $<100$ & & & & \\
\hline 147-895D- & & & & & & & \\
\hline $2 \mathrm{R}-134-38$ & 16.34 & Basalt & $<100$ & & & - & - \\
\hline $3 \mathrm{R}-1,108-111$ & 27.08 & Harzburgite & $<100$ & & & & \\
\hline $3 \mathrm{R}-1,133-139$ & 27.33 & Harzburgite & 200 & & & & \\
\hline $4 \mathrm{R}-3,44-46$ & 37.43 & Harzburgite (serp.) & $<100$ & & & - & - \\
\hline $4 R-3,50-56$ & 37.49 & Harzburgite (serp.) & $<100$ & & & & \\
\hline $4 \mathrm{R}-4,87-91$ & 39.32 & Harzburgite (serp.) & 300 & & & & \\
\hline $5 \mathrm{R}-1,50-52$ & 43.80 & Harzburgite & $<100$ & & & - & - \\
\hline $6 \mathrm{R}-1,128-134$ & 56.30 & Harzburgite & 170 & & & & \\
\hline $7 \mathrm{R}-1,23-27$ & 64.83 & Gabbro & 240 & & & & \\
\hline $7 \mathrm{R}-1,41-43$ & 65.01 & Troctolite & $<100$ & & & & \\
\hline $7 \mathrm{R}-1,45-49$ & 65.05 & Troctolite & 1390 & 99.9 & 0.1 & +0.3 & - \\
\hline $7 \mathrm{R}-1,65-67$ & 65.25 & Troctolite & 400 & & & & \\
\hline $7 \mathrm{R}-1,74-79$ & 65.34 & Troctolite & 490 & & & & \\
\hline $7 R-1,131-135$ & 65.91 & Harzburgite (serp.) & 270 & & & - & - \\
\hline $7 \mathrm{R}-2,8-12$ & 66.20 & Harzburgite (serp.) & 350 & & * & & \\
\hline $8 \mathrm{R}-2,123-125$ & 76.91 & Dunite (serp.) & 700 & & & -23.7 & - \\
\hline $8 R-2,132-135$ & 77.00 & Dunite (serp.) & 500 & & & -26.3 & - \\
\hline
\end{tabular}


Table 2 (continued).

\begin{tabular}{|c|c|c|c|c|c|c|c|}
\hline \multirow[b]{2}{*}{$\begin{array}{l}\text { Core, section, } \\
\text { interval }(\mathrm{cm})\end{array}$} & \multirow[b]{2}{*}{$\begin{array}{l}\text { Depth } \\
\text { (mbsf) }\end{array}$} & \multirow[b]{2}{*}{ Lithology } & \multirow{2}{*}{$\begin{array}{l}\text { Sulfur } \\
\text { content } \\
\text { (ppm) }\end{array}$} & \multicolumn{2}{|c|}{$\%$ of $S$ fixed as } & \multirow{2}{*}{$\frac{\delta^{2 / S} S}{\text { AVS }}$} & \multirow{2}{*}{$\frac{\delta^{24} \mathrm{~S}}{\text { Pyrite }}$} \\
\hline & & & & Avs & Pyrite & & \\
\hline \multicolumn{8}{|l|}{ 147-895E- } \\
\hline IR-1, 64-68 & 0.64 & Olivine gabbro & $<100$ & & & & \\
\hline IR $-3,20-24$ & 3.06 & Harzburgite (serp.) & $<100$ & & & & \\
\hline IR-3,51-55 & 3.37 & Dunite (serp.) & 1200 & & & & \\
\hline IR-3, 62-64 & 3.48 & Harzburgite (serp.) & $<100$ & & & & \\
\hline IR-3, 65-66 & 3.51 & Dunite (serp.) & 300 & 94.7 & 5.3 & -3.3 & - \\
\hline IR- $-3,74-76$ & 3.60 & Dunite (serp.) & 390 & & & & \\
\hline IR-4, 28-32 & 4.60 & Dunite (serp.) & 560 & & & +0.6 & - \\
\hline $3 R-2,39-46$ & 31.36 & Dunite (serp.) & 425 & & & & \\
\hline $3 \mathrm{R}-2,89-91$ & 31.86 & Troctolite & 300 & 77.2 & 22.8 & +2.5 & -4.8 \\
\hline $4 \mathrm{R}-1,29-31$ & 39.79 & Troctolite & 400 & & & & \\
\hline $4 \mathrm{R}-1,34-36$ & 39.84 & Troctolite & 1750 & & & & \\
\hline $4 R-I, 117-126$ & 40.67 & Harzburgite (serp.) & 510 & & & & \\
\hline $4 \mathrm{R}-1,131-134$ & 40.81 & Harzburgite (serp.) & 500 & & & & \\
\hline $4 \mathrm{R}-1,135-137$ & 40.85 & Harzburgite (serp.) & 700 & & & -6.5 & -1.6 \\
\hline $4 \mathrm{R}-2,18-2 \mathrm{I}$ & 41.15 & Dunite & 260 & & & & \\
\hline $4 \mathrm{R}-2,105-109$ & 42.05 & Dunite & 200 & & & & \\
\hline $5 \mathrm{R}-1,31-33$ & 49.21 & Olivine gabbro & $<100$ & & & - & - \\
\hline $6 \mathrm{R}-1,82-84$ & 59.42 & Troctolite & 1100 & 2.8 & 97.2 & - & +7.8 \\
\hline $6 \mathrm{R}-1,90-92$ & 59.50 & Troctolite & 330 & & & & \\
\hline $6 \mathrm{R}-1,110-114$ & 59.70 & Dunite (serp.) & 500 & 98.9 & 1.1 & -29.3 & - \\
\hline $6 \mathrm{R}-3,89-95$ & 62.20 & Dunite & 170 & & & & \\
\hline $6 \mathrm{R}-4,33-37$ & 62.73 & Dunite & 280 & & & & \\
\hline $7 R-1,123-128$ & 69.48 & Dunite & $<100$ & & & & \\
\hline $7 \mathrm{R}-2,44-49$ & 70.14 & Dunite & 200 & & & & \\
\hline $7 \mathrm{R}-4,48-51$ & 73.05 & Harzburgite (serp.) & 220 & & & & \\
\hline $8 \mathrm{R}-3,39-44$ & 81.30 & Dunite & 270 & & & & \\
\hline $8 R-4,28-35$ & 82.58 & Dunite & 400 & & & & \\
\hline \multicolumn{8}{|l|}{$147-895 \mathrm{~F}$ - } \\
\hline IR-1, 1-4 & 0.01 & Harzburgite (serp.) & 200 & & & & \\
\hline $2 \mathrm{R}-1.65-69$ & 18.85 & Harzburgite (serp.) & $<100$ & & & & \\
\hline $2 \mathrm{R}-2,66-71$ & 20.36 & Harzburgite (serp.) & 200 & & & & \\
\hline
\end{tabular}

Notes: AVS = acid-volatile sulfides; values in parentheses have a higher error; $-=$ sulfur recovery too low for isotope measurement.

Table 3. Average, minimum, and maximum sulfur contents (ppm), grouped according to lithology.

\begin{tabular}{lrrrr}
\hline \multicolumn{1}{c}{ Rock type } & $\begin{array}{c}\text { No. of } \\
\text { samples }\end{array}$ & Mean & Minimum & Maximum \\
\hline Basalt & 7 & 620 & $<100$ & 1020 \\
Dunite (total) & 16 & 400 & $<100$ & 1200 \\
Dunite (less altered) & 8 & 230 & $<100$ & 400 \\
Dunite (serpentinized) & 8 & 570 & 300 & 1200 \\
Gabbro & 14 & 1590 & $<100$ & 10340 \\
Gabbronorite & 39 & 760 & $<100$ & 3100 \\
Harzburgite (total) & 20 & 200 & $<100$ & 700 \\
Harzburgite (less altered) & 5 & 100 & $<100$ & 200 \\
Harzburgite (serpentinized) & 15 & 250 & $<100$ & 700 \\
Troctolite & 9 & 680 & $<100$ & 1700 \\
\hline
\end{tabular}

the rocks of Hole $895 \mathrm{E}$. Plagioclase is replaced here by a fibrous intergrowth of tremolite-prehnite, amphibole, and clay minerals. The dark green olivines are transformed into talc, amphibole, chlorite, serpentine, and sporadic native copper.

Of the samples obtained for investigation, only seven are basalts. They belong to the dikes found in Holes 894G, 895A, and 895D. The sulfur content of these ranges from less than 100 to $1020 \mathrm{ppm}$ and averages $620 \mathrm{ppm}$. These values are close to results obtained in earlier investigations on oceanic basalts (e.g., Andrews, 1979; Puchelt and Hubberten, 1980; Sakai et al., 1982; Hubberten and Puchelt, 1985; Alt et al., 1989; Alt and Anderson, 1991; Alt, 1994).

\section{Sulfur Isotopes}

Sulfur isotope ratios were determined separately for AVS and pyrite. The results are presented in Table 2. In most samples, AVS as well as pyrite were present. However, because of small sample sizes and/or low S contents, it was sometimes impossible to obtain sufficient sulfur (as CdS) for isotope analysis. Isotope ratios for the total (bulk) sulfidic sulfur were evaluated by balancing the $\delta^{34} \mathrm{~S}$ values of the two fractions (AVS and pyrite) with the relative amount of each.
The distribution of these values are displayed in Figure 3, according to lithology.

\section{Hole $894 G$}

In Hole $894 \mathrm{G}$, a sulfur isotope profile over $140 \mathrm{~m}$ of lower oceanic crust was obtained. No systematic, depth-related trends were observed here. The bulk $\delta^{34} \mathrm{~S}$ values in the gabbroic rocks (gabbronorites and gabbros) cover a range between $-2.4 \%$ and $+6.4 \%$, with an average of $+2.2 \%$. The means are quite similar for the AVS and pyritic fractions $(+1.5 \%$ and $+2.0 \%$ ) , but the individual data sets show considerably higher dispersion relative to the bulk sulfidic $\delta^{34} \mathrm{~S}$ values (between $-9 \% \circ$ and $+9 \%$ ). In many samples, pyrite sulfur is depleted in ${ }^{34} \mathrm{~S}$ compared to the AVS, which points to an isotopic disequilibrium. These isotopic data are extremely close to those of comparable sequences of the lower oceanic crust. The Layer 3 gabbros recovered at Hole $735 \mathrm{~B}$ display slightly lower values $(+0.8 \% \pm 0.9 \%$ ), but sequences that underwent subsequent hydrothermal alteration are biased toward more positive values (up to $+6.9 \%$ ) (Alt and Anderson, 1991). In the lower gabbros of the Troodos ophiolitic crust, $\delta^{34} \mathrm{~S}$ ranges from $+0.1 \%$ to $+5.9 \%$ (Alt, 1994), and in the Shetland ophiolite it averages $+3.3 \%$ (Maynard, 1993).

\section{Site 895}

The low sulfur content and small sample size of the mantle-derived rocks recovered at Site 895 did not permit determination of a systematic $\mathrm{S}$ isotope profile. Because monosulfides dominate in dunites and harzburgites, only the AVS fraction could be analyzed. The six values measured in these rocks cluster into two groups: three of them are in a slightly negative range, but still close to $0 \% 0(+0.6 \%$, $-3.3 \%$, and $-4.1 \%$ ), whereas the other three are related to highly serpentinized dunites and strongly depleted in ${ }^{34} \mathrm{~S}(-23.7 \%$, $-26.3 \%$, and $-29.3 \%$ ). Two of the troctolite samples yielded $\delta^{34} \mathrm{~S}$ values for total sulfur close to $0 \% o(0.3 \% o$ and $0.8 \% o)$, and one is quite enriched in ${ }^{34} \mathrm{~S}(+7.8 \%$ ) 


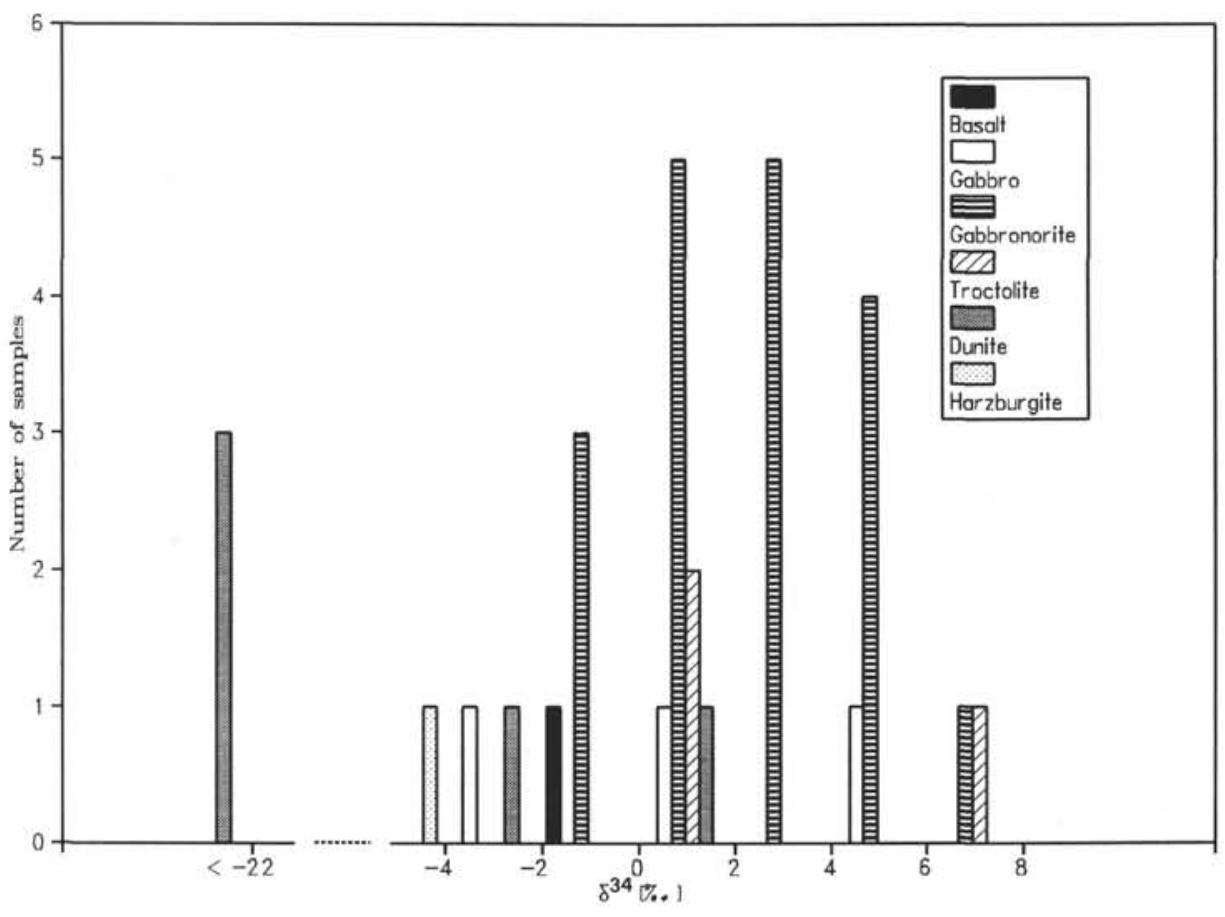

Figure 3. Distribution of bulk $\delta^{34} \mathrm{~S}$ values in samples with various lithologies.

\section{DISCUSSION}

\section{Site 894}

Our data on the sulfide mineralogy, sulfur content, and isotopic composition of the profile sampled at Site 894 generally agree with results of previous investigations on the lower oceanic crust (Alt and Anderson, 1991; Maynard, 1993; Alt, 1994).

Although primary microstructural features are blurred to some extent by later stage alteration, they suggest that a paragenesis of pyrrhotite \pm chalcopyrite may have crystallized from an immiscible $\mathrm{Ni}$ $\mathrm{Fe}-\mathrm{Cu}$ sulfide liquid separated at the early stages of mafic magma evolution. The high $\mathrm{Ni}$ contents in the analyzed pyrrhotites suggest that crystallization preceded $\mathrm{Ni}$ extraction from the magma by olivine (Fleet and MacRae, 1987). The Ni concentration in the sulfide melt, on the other hand, was not high enough to permit crystallization of pentlandite, which was found mostly as a secondary phase. Not only the microscopic features but also the high $\mathrm{Ni}$ contents in pyrite suggest that some of the pyrite results from the alteration of such pyrrhotites (Table 1). The secondary sulfide assemblage (pyrite \pm marcasite \pm chalcopyrite \pm pentlandite \pm sphalerite) is typically localized in zones of intensive alteration. The higher sulfur content of these sequences clearly indicates an external sulfur input. From a profile through Hole 894G (Fig. 4), it is evident that high sulfur concentrations are coupled mostly with an increase of the pyritic (marcasitic) fraction, although high pyrite contents are also associated with low S contents, too (in these samples, mostly secondary after pyrrhotite).

The primary sulfur content of the investigated gabbroic rocks is related to the sulfur concentration of the basic melt, which in turn is a function of pressure, temperature, and, in particular, iron content and the fugacity of oxygen and sulfur (Wallace and Carmichael, 1992). Strong alteration of the primary composition by late metamorphic events makes it impossible to trace back the evolution of sulfur contents by applying the quantitative relationships established by these authors. They considered that preeruptive $\mathrm{S}$ concentrations in basaltic magmas must have been between 800 and $2500 \mathrm{ppm}$. According to Mathez (1976), basaltic melts can contain up to $1500 \mathrm{ppm}$ sulfur depending on the iron content, and the $\mathrm{S}$ content of primitive mid-oceanic ridge basalt (MORB) magmas resulting by partial melting of $10 \%-20 \%$ of a mantle source with $80-300 \mathrm{ppm}$ of $\mathrm{S}$ is restricted to about $800-1000$ ppm (Chaussidon et al., 1989). These values can therefore be taken as the upper limits of the primary sulfur contained in the investigated rocks. However, considering the effects of the metamorphic processes on the distribution of sulfur, it is reasonable to start from the mean of the gabbroic rocks at Hole 894G (i.e., $760 \mathrm{ppm}$ ), which is well within the range of primary igneous sulfur concentrations given by Alt and Anderson (1991) for Layer 3 gabbros in Hole 735B $(610 \pm 180 \mathrm{ppm})$.

A depletion in sulfur during the early stages of dynamothermal metamorphism and the subsequent static hydrothermal metamorphism cannot be excluded and would be responsible for the very low values $(<100 \mathrm{ppm})$ found in some of the samples. However, the mobilization of $\mathrm{S}$ was probably restricted to a relative small scale. A more intensive circulation of seawater-related fluids occurred in later episodes of alteration, leading to an enrichment through incorporation of sulfides derived from reduction of seawater sulfates.

The isotopic signature of the gabbroic rocks at Site $894(+2.2 \%$ ), on the whole, is slightly higher than the generally accepted $0 \%$ of the magmatic (MORB) sulfur reservoir. Variations in the primary isotopic composition of the source are considered low. Possible causes are equilibrium fractionation related to the speciation of sulfur as a function of oxygen and sulfur fugacities or magmatic differentiation (Ueda and Sakai, 1984).

The similar isotopic signatures of the AVS and pyrite fractions, with means close to $0 \%$, suggest to a first approximation the same (possibly magmatic) sulfur source for both. Nevertheless, although pyrite and marcasite must have formed at temperatures below $742^{\circ} \mathrm{C}$ (Barton and Skinner, 1979; Barker and Parks, 1986), a portion of the AVS in the analyzed samples might have a primary magmatic origin. A low-temperature breakdown of a monosulfide solid solution can also lead to an symplectic intergrowth of "primary" pyrite + pentlandite (Chaussidon and Lorand, 1990). However, a part of the bisulfides (pyrite) is derived from the in situ alteration of primary sulfides (pyrrhotite), as microscopic investigations demonstrate. In this case, the sulfur keeps its original magmatic isotopic signature. 


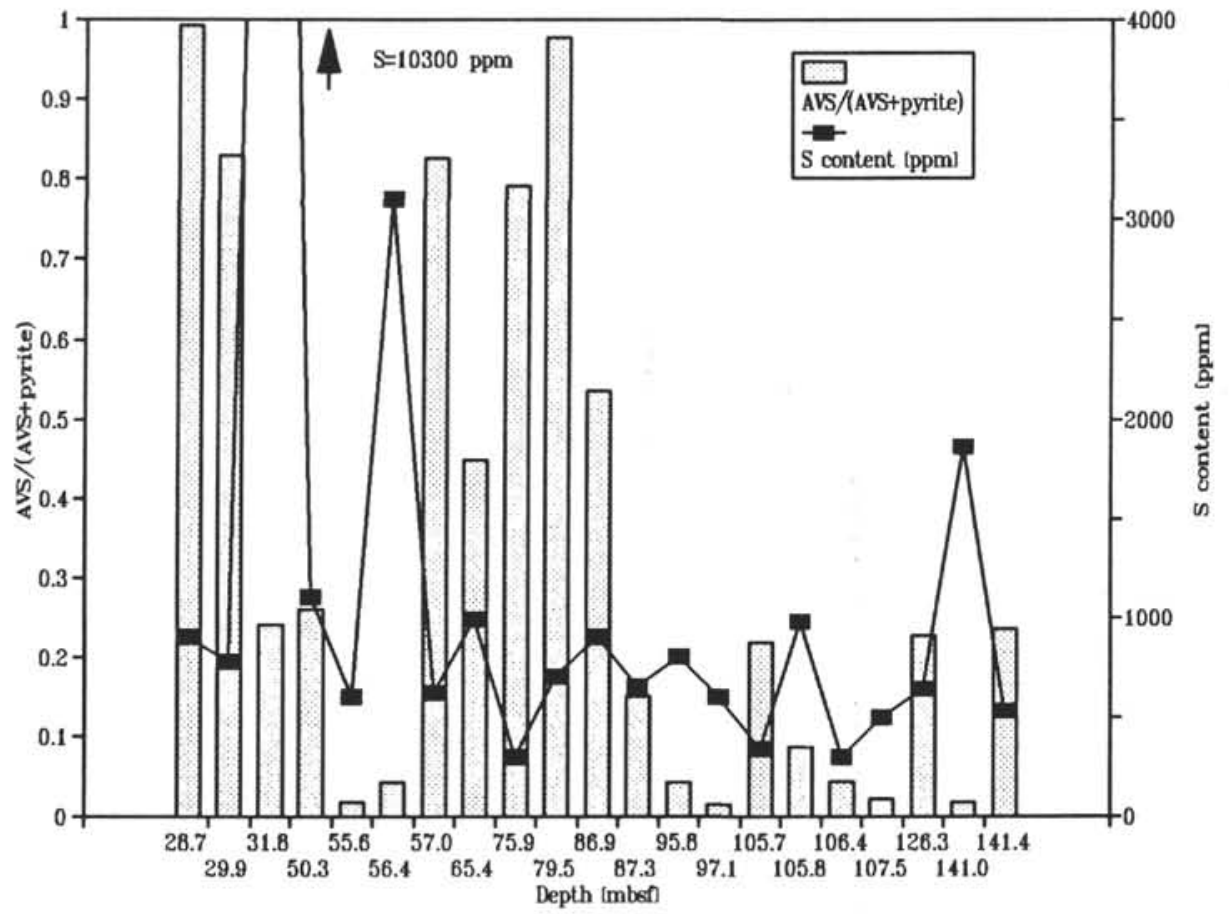

Figure 4. Variation of sulfur content and AVS/(AVS + pyrite) ratio with depth in Hole 894G.

Nevertheless, the relatively wide range of variation and the light positive shift of the average of the $\delta^{34} \mathrm{~S}$ values suggest that other sources must be considered in addition to magmatic sulfur. In some of the samples, the depletion of pyrite sulfur in ${ }^{34} \mathrm{~S}$ as compared to the AVS points to an isotopic disequilibrium and, as a consequence, suggests different mechanisms and episodes of formation.

If present-day seawater $\left(\delta^{34} \mathrm{~S}=+20.9 \%\right.$; Rees et al., 1978) is considered as a source for this external sulfur, we have to find a reduction mechanism that at the same time explains the isotopic shift from the $\delta^{34} \mathrm{~S}$ value of the seawater sulfate to the values measured in sulfides. Under the actual conditions, an "abiogenic" mechanism seems plausible. At the temperature of hydrothermal fluids $\left(>200^{\circ} \mathrm{C}\right.$; Ohmoto, 1986), the equilibrium fractionation between sulfide and sulfate alone could produce $\delta^{34} \mathrm{~S}$ values for sulfides with a "magmatic" $0 \%$ signature. The fractionation factor is a function of temperature, oxygen fugacity, $\mathrm{pH}$, and ionic strength. Generally, inorganic sulfate reduction at temperatures below $200^{\circ} \mathrm{C}$ is kinetically inhibited and becomes reasonable in hydrothermal systems only above this temperature (Ohmoto and Lasaga, 1982). The possibility of inorganic sulfate reduction was discussed by Shanks et al. (1981). They proposed a reaction in which $\mathrm{Fe}^{2+}$ from fayalite forms pyrite, magnetite, and hematite. Ferrous iron is oxidized to reduce seawater sulfate, which is then incorporated into the fluids and rocks and results in higher $\delta^{34} \mathrm{~S}$ values of the sulfide.

Andrews (1979) proposed a model in which oxidation of sulfides under limited oxygen supply leads to the formation of sulfite and thiosulfate. The spontaneous disproportionation of these unstable species leads to a depletion of ${ }^{34} \mathrm{~S}$ in sulfides formed from such solutions and could account for a shift to more negative $\delta^{34} S$ values.

\section{Site 895}

In the mantle-derived peridotitic sequences at Site 895, pentlandite and chalcopyrite are the dominant primary sulfides, and secondary Ni-sulfides, native metals, and alloys (copper, awaruite) occur as alteration products. The lower abundance of primary pyrrhotite is in agreement with the presence of a refractory peridotite residue (i.e., with the moderately to highly depleted character of the rocks in Hess Deep). Fe-sulfides (pyrrhotite) are restricted mostly to relatively primitive peridotites because iron tends to enter the melt preferentially, leaving the residual solids relatively enriched in $\mathrm{Ni}$ and $\mathrm{Cu}$ (Mitchell and Keays, 1981; Garuti et al., 1984).

The presence of awaruite and native copper is significant and casts some light on the conditions in which alteration of the primary assemblages took place. Awaruite (an alloy in the compositional range $\mathrm{Ni}_{2} \mathrm{Fe}$ and $\mathrm{Ni}_{3} \mathrm{Fe}$ ) in terrestrial conditions has been reported in association with serpentinized peridotites from about 20 localities (Rubin, 1991). The common occurrence of awaruite together with native metals, and the appearance of $\mathrm{H}_{2}$ gas in springs draining areas of active serpentinization, suggests strongly reducing conditions. These are related to mineral reactions leading to $\mathrm{H}_{2}$ as a reaction product such as (Frost, 1985):

$$
\begin{aligned}
& 6 \mathrm{Fe}_{2} \mathrm{SiO}_{4}+7 \mathrm{H}_{2} \mathrm{O}=3 \mathrm{Fe}_{3} \mathrm{Si}_{2} \mathrm{O}_{5}(\mathrm{OH})_{4}+\mathrm{Fe}_{3} \mathrm{O}_{4}+\mathrm{H}_{2} \\
& \text { olivine serpentine magnetite }
\end{aligned}
$$

and subsequently to formation of awaruite (Bird and Weathers, 1979):

$$
2 \mathrm{FeNiS}{ }_{2}+3 \mathrm{H}_{2}=\mathrm{Ni}_{2} \mathrm{Fe}+\mathrm{FeS}+3 \mathrm{H}_{2} \mathrm{~S} .
$$

Phase relations among Fe-Ni sulfides in serpentinites led Frost (1985) to the conclusion that the appearance of awaruite is favored by high $\mathrm{Ni}$ and low $\mathrm{S}$ abundances. In consequence, fluids involved in the formation of awaruite must have been low in sulfur and contributed to the leaching of sulfur (on a local scale?) from the rocks during serpentinization.

Because of its incompatible behavior, the sulfur content of the mantle rocks is inversely correlated with the degree of partial melting and magma extraction (Sun, 1982; Lorand, 1989; Ionov et al., 1992). High $\mathrm{Mg}$ values and low abundances of clinopyroxene could therefore be considered to indicate that the rocks did not undergo significant sulfur fractionation. Their sulfur content is representative for the 
mantle source. If we agree with Chaussidon and Lorand (1990) that during serpentinization only a restricted and localized remobilization of sulfur occurs, based on the average content of the peridotitic rocks at Site 895 , we derive an abundance of about $300 \mathrm{ppm} \mathrm{S}$ for the mantle. This is close to the range of values measured in orogenic-type peridotites. The discrepancy with the considerably lower sulfur observed in mantle xenoliths (by a factor of up to 10) is accounted for by the loss caused by low-temperature alteration (secondary oxyhydration) of pristine magmatic sulfides or their replacement by magnetite (Mitchell and Keays, 1981; Lorand, 1990).

Although there is a general consensus that the average isotopic composition of sulfur in the mantle at about $0 \% \circ \pm 2 \%$ is close to the meteoric value, the relevant literature data are much more scattered (Schneider, 1970; Hubberten, 1984; Kyser, 1986; Chaussidon et al., 1987, 1989; Chaussidon and Lorand, 1990; Ionov et al., 1992). Chaussidon and Lorand (1990) were the first to report negative $\delta^{34} \mathrm{~S}$ values in mantle peridotites and relate them to an early depletion event in the upper mantle. Heterogeneity is explained by postulating two extreme reservoirs with different isotopic compositions at about $-3 \%$ and $+3 \% \circ$ in the continental upper mantle. Subduction of crustal slabs enriched in ${ }^{34} \mathrm{~S}$ and isotopic fractionation between residual peridotites and generated basaltic melts are further mechanisms invoked to explain the relative large interval in which the $\delta^{34} \mathrm{~S}$ values of mantle-derived rocks fall (Ueda and Sakai, 1984; Harmon et al., 1987; Ionov et al., 1992; Maynard, 1993). The group of slightly negative $\delta^{34} \mathrm{~S}$ values in the dunites and harzburgites at Site 895 is similar with those found by Chaussidon and Lorand (1990), although this comparison to the subcontinental mantle is not rigorous in many respects.

Three samples of serpentinized dunites are strongly depleted in ${ }^{34} \mathrm{~S}\left(\delta^{34} \mathrm{~S}\right.$ values between $-23.7 \%$ and $-29.3 \%$ ) . Far higher, but still relatively low, is a $\delta^{34} \mathrm{~S}$ value $(-10.1 \%$ ) found in the serpentinized lherzolite massif from Ariege (Northeastern Pyrenees, France) by the same authors. Such negative $\delta^{34} \mathrm{~S}$ values occurring in this environment are yet not well understood. Their association with intensely serpentinized rocks could hint at a possible genetic relationship with the strongly reducing conditions that prevail during serpentinization. Maynard (1993) considered the possible isotopic significance of the reduction of sulfides to awaruite during the serpentinization of Shetland dunites, based on an equilibrium fractionation. Assuming a Rayleigh distillation model, he concluded that only an insignificant fractionation could occur. Rather, kinetic fractionation is more probable. The slower reaction kinetic of ${ }^{34} \mathrm{~S}$ would lead to an isotopically light $\mathrm{H}_{2} \mathrm{~S}$, which will produce at some distance a secondary sulfide phase depleted in ${ }^{34} \mathrm{~S}$. Experimental studies are necessary to determine the kinetic fractionation effect caused by the reduction of sulfides by hydrogen.

\section{CONCLUSIONS}

New data on the geochemistry of sulfur were obtained on samples recovered from Hess Deep, eastern Pacific Ocean, during ODP Leg 147. Two distinct sections of oceanic lithosphere were investigated: a profile through the lower part of the oceanic crust at Site 894 and a transition zone to the upper residual mantle at Site 895.

In the gabbroic rocks at Site 894, the primary sulfidic paragenesis pyrrhotite \pm chalcopyrite is not well preserved and is widely replaced by a secondary sulfide assemblage. However, the slight positive shift of the $\mathrm{S}$ isotope data from a primary signature of about $0 \%$ appears to suggest a restricted external, seawater-derived sulfur input. As a result, we conclude that, although the primary sulfur content was redistributed during the subsequent alteration, the average of the gabbroic rocks of $760-780 \mathrm{ppm}$ is still quite close to the primary sulfur abundance in the lower oceanic crust.

The sulfide assemblage in the mantle-derived peridotitic rocks at Site 895 is dominated by Ni-rich phases (mainly pentlandite) and by their alteration products, rather than pyrrhotite. Alteration and mobilization of sulfur, as native $\mathrm{Cu}$ and $\mathrm{Ni}-\mathrm{Fe}$ alloys show, occurred under the strongly reducing conditions of serpentinization. However, this mobilization was restricted, and had only a local character. On the other hand, we can exclude a significant change in the pristine sulfur content that might have been caused by the fractionation of sulfur during magma extraction, based on petrological considerations. We therefore consider that the average sulfur content of the peridotitic rocks at Site 895 (about $300 \mathrm{ppm}$ ) reflects quite well the initial sulfur content in the mantle source. The isotopic data obtained are too scarce and too broadly scattered to permit valid conclusions. We relate the three very low $\delta^{34} \mathrm{~S}$ values obtained at this site to the strongly reducing conditions that prevailed during serpentinization.

\section{ACKNOWLEDGMENTS}

We are grateful to the Ocean Drilling Program for permitting two of us (H.P. \& H.M.P) to participate in Leg 147. We would like to thank all scientists on board JOIDES Resolution and at the postcruise meeting for interesting discussions and helpful suggestions. We (H.P. \& Z.B.) thank the Deutsche Forschungsgemeinschaft for funding our participation in the cruise and in subsequent research work and H.M.P. was funded by a Royal Society fellowship during the period of this research. We are grateful to J. Alt and L. Woodruff and an unknown editorial reviewer for valuable suggestions leading to a considerable improvement of the manuscript.

\section{REFERENCES}

Alt, J.C., 1994. A sulfur isotopic profile through the Troodos ophiolite, Cyprus: primary composition and the effects of seawater hydrothermal alteration. Geochim. Cosmochim. Acta, 58:1825-1840.

Alt, J.C., and Anderson, T.F., 1991. Mineralogy and isotopic composition of sulfur in layer 3 gabbros from the Indian Ocean, Hole 735B. In Von Herzen, R.P., Robinson, P.T., et al., Proc. ODP, Sci. Results, 118: College Station, TX (Ocean Drilling Program), 113-126.

Alt, J.C., Anderson, T.F., and Bonnell, L., 1989. The geochemistry of sulfur in a $1.3 \mathrm{~km}$ section of hydrothermally altered oceanic crust, DSDP Hole 504B. Geochim. Cosmochim. Acta, 53:1011-1023.

Andrews, A.J., 1979. On the effect of low-temperature seawater-basalt interaction on the distribution of sulfur in oceanic crust, Layer 2. Earth Planet. Sci. Lett., 46:68-80.

Barker, W.W., and Parks, T.C., 1986. The thermodynamic properties of pyrrhotite and pyrite: a re-evaluation. Geochim. Cosmochim. Acta, 50:21852194.

Barton, P.B., Jr., and Skinner, B.J., 1979. Sulfide mineral stabilities. In Barnes, H.L. (Ed.), Geochemistry of Hydrothermal Ore Deposits (2nd ed.): New York (Wiley), 278-403.

Berner, Z., Blum, N., and Puchelt, H., 1993. Sulfur isotope geochemistry of the KTB pilot hole crustal transect, Upper Palatine, Germany. Sci. Drill., 4:23-29.

Bird, J.M., and Weathers, M.S., 1979. Origin of josephinite. Geochem. J., 13:41-55.

Chaussidon, M., Albarede, F., and Sheppard,S.M.F., 1987. Sulphur isotope heterogeneity in the mantle from ion microprobe measurements of sulphide inclusions in diamonds. Nature, 330:242-244.

1989. Sulphur isotope variations in the mantle from ion microprobe analyses of micro-sulphide inclusions. Earth Planet. Sci. Lett. 92:144-156.

Chaussidon, M., and Lorand, J.P., 1990. Sulphur isotope composition of orogenic spinel lherzolite massifs from Ariege (north-eastern Pyrenees, France): an ion microprobe study. Geochim. Cosmochim. Acta, 54:28352846.

Fleet, M.E., and MacRae, N.D., 1987. Partition of Ni between olivine and sulfide: the effect of temperature, $\mathrm{f}_{\mathrm{O} 2}$ and $\mathrm{f}_{\mathrm{S} 2}$. Contrib. Mineral. Petrol., 95:336-342.

Frost, B.R., 1985. On the stability of sulfides, oxides, and native metals in serpentine. J. Petrol., 26:31-63.

Garuti, G., Gorgoni, C., and Sighinolfi, G.P., 1984. Sulfide mineralogy and chalcophile element abundances in the Ivrea-Verbano mantle peridotites (Western Italian Alps). Earth Planet. Sci. Lett., 70:69-87.

Harmon, R.S., Hoefs, J., and Wedepohl, K.H., 1987. Stable isotope (O, H, S) relationships in Tertiary basalts and their mantle xenoliths from the 
Northern Hessian Depression, W. Germany. Contrib. Mineral. Petrol., 95:350-369.

Hartmann, G., and Wedepohl, K.H.. 1990. Metasomatically altered peridotite xenoliths from the Hessian Depression (Northwest Germany). Geochim. Cosmochim. Acta, 54:71-86.

Hubberten, H.W., 1984. Die Fraktionierung der Schwefelisotope bei der Entstehung und Veränderung der ozeanischen Kruste. Dr. Habil [Thesis]. Univ. of Karlsruhe, Germany.

Hubberten, H.W., and Puchelt, H., 1985. The sulfur content and isotopic composition in basalts from holes 558, 559, 562, and 564. In Bougault, H., Cande, S.C., et al. (Eds.), Init. Repts. DSDP, 82: Washington (U.S. Govt. Printing Office), 519-522.

Ionov, D.A., Hoefs, J., Wedepohl, K.H., and Wiechert, U., 1992. Content and isotopic composition of sulphur in ultramafic xenoliths from central Asia. Earth Planet. Sci. Lett., 111:269-286.

Kovalenko, V.I., Solovova, I.P., Naumov, V.B., Ryabchikov, D.A., Ionov, D.A., and Bogatikov, O.A., 1987. Fluidized $\mathrm{CO}_{2}$-sulphide-silicate media as agents of mantle metasomatism and megacryst formation: evidence from large druse in a spinel-lherzolite xenolith. Phys. Earth Planet. Inter., 45:280-293.

Kyser, T.K., 1986. Stable isotope variations in the mantle. In Valley, J.W., Taylor, H.P., and O'Neil, J.R. (Eds.), Stable Isotopes in High Temperature Geological Processes: Miner. Soc. Am., Rev. Mineral., 16:157-178.

Lorand, J.P., 1989. Abundance and distribution of Cu-Fe-Ni sulfides, sulfur, copper and platinum-group elements in orogenic-type spinel lherzolite massifs of Ariege (north-eastern Pyrenees, France). Earth Planet. Sci. Lett., 93:50-64.

, 1990. Are spinel therzolite xenoliths representative of the abundance of sulfur in the upper mantle? Geochim. Cosmochim. Acta, 54:1487-1492.

, 1991. Sulphide petrology and sulphur geochemistry of orogenic Iherzolites: a comparative study of the Pyrenean bodies (France) and the Lanzo Massif (Italy), In Orogenic Lherzolites and Mantle Processes. $J$. Petrol. Spec. Vol., 77-96.

Mathez, E.A., 1976. Sulfur solubility and magmatic sulfides in submarine basalt glass. J. Geophys. Res., 81:4269-4276.

Maynard, J., 1993. The genesis of sulphides in the Shetland Ophiolite [Ph.D. dissert.]. The Open Univ., Milton Keynes, United Kingdom.

Mével, C., and Cannat, M., 1991. Lithospheric stretching and hydrothermal processes in oceanic gabbros from slow-spreading ridges. In Peters, T., Nicolas, A., and Coleman, R.G. (Eds.), Ophiolite Genesis and Evolution of the Oceanic Lithosphere: Dordrecht (Kluwer), 293-312.

Mével, C., Gillis, K.M., and Shipboard Scientific Party, 1993. Introduction and principal results. In Gillis, K., Mével, C., Allan, J., et al., Proc. $O D P$, Init. Repts., 147: College Station, TX (Ocean Drilling Program), 5-14.

Mitchell, R.H., and Keays, R.R., 1981. Abundance and distribution of gold, palladium and iridium in some spinel and garnet lherzolites: implications for the nature and origin of precious metal-rich intergranular components in the upper mantle. Geochim. Cosmochim. Acta, 45:2425-2442.

Ohmoto, H., 1986. Stable isotope geochemistry of ore deposits. In Valley, J.W., Taylor, H.P., Jr., and O'Neil, J.R. (Eds.), Stable Isotopes in High Temperature Geological Processes. Miner. Soc. Amer., Rev. Mineral., $16: 491-559$

Ohmoto, H., and Lasaga, A.C., 1982. Kinetics of reactions between aqueous sulfates and sulfides in hydrothermal systems. Geochim. Cosmochim. Acta, 46:1727-1745.

Puchelt, H., and Hubberten, H.-W., 1980. Preliminary results of sulfur isotope investigations on Deep Sea Drilling Project cores from Legs 52 and 53. In Donnelly, T., Francheteau, J., Bryan, W., Robinson, P., Flower, M., Salisbury, M., et al., Init. Repts. DSDP, 51, 52, 53 (Pt. 2):Washington (U.S. Govt. Printing Office), 1145-1148.

Rees, C.E., Jenkins, W.J., and Monster, J., 1978. The sulphur isotopic composition of ocean water sulphate. Geochim. Cosmochim. Acta, 42:377381.

Ringwood, A.E., and Kesson, S.E., 1976. Basaltic magmatism and the composition of the moon, II. Siderophile and volatile elements in the moon, earth and chondrites: implications for lunar origin. Publ.-Aust. Natl. Univ., Res. Sch. Earth Sci., 1222.

Rubin, A.E., 1991. Euhedral awaruite in the Allende meteorite: implications for the origin of awaruite-and magnetite-bearing nodules in $\mathrm{CV} 3$ chondrites. Am. Mineral., 76:1356-1362.

Sakai, H., Casadevall, T.J., and Moore, J.G., 1982. Chemistry and isotope ratios of sulfur in basalts and volcanic gases at Kilauea volcano, Hawaii. Geochim. Cosmochim. Acta, 46:729-738.

Schneider, A., 1970. The sulfur isotope composition of basaltic rocks. Contrib. Mineral. Petrol., 25:95-124.

Shanks, W.C., III, Bischoff, J.L., and Rosenbauer, R.J., 1981. Seawater sulfate reduction and sulfur isotope fractionation in basaltic systems: interaction of seawater with fayalite and magnetite at $200-350^{\circ} \mathrm{C}$. Geochim. Cosmochim. Acta, 45:1977-1995.

Sun, S., 1982. Chemical composition and origin of the Earth's primitive mantle. Geochem. Cosmochim. Acta, 46:179-192.

Thode, H.G., Monster, J., and Dunford, H.B., 1961. Sulphur isotope geochemistry. Geochim. Cosmochim. Acta, 25:158-174.

Ueda, A., and Sakai, H., 1984. Sulfur isotope study of Quaternary volcanic rocks from the Japanese Islands Arc. Geochim. Cosmochim. Acta, 48:1837-1848.

Wallace, P., and Carmichael, I.S.E., 1992. Sulfur in basaltic magmas. Geochim. Cosmochim. Acta, 56:1863-1874.

Date of initial receipt: 5 August 1994

Date of acceptance: 12 January 1995

Ms 147SR-005 

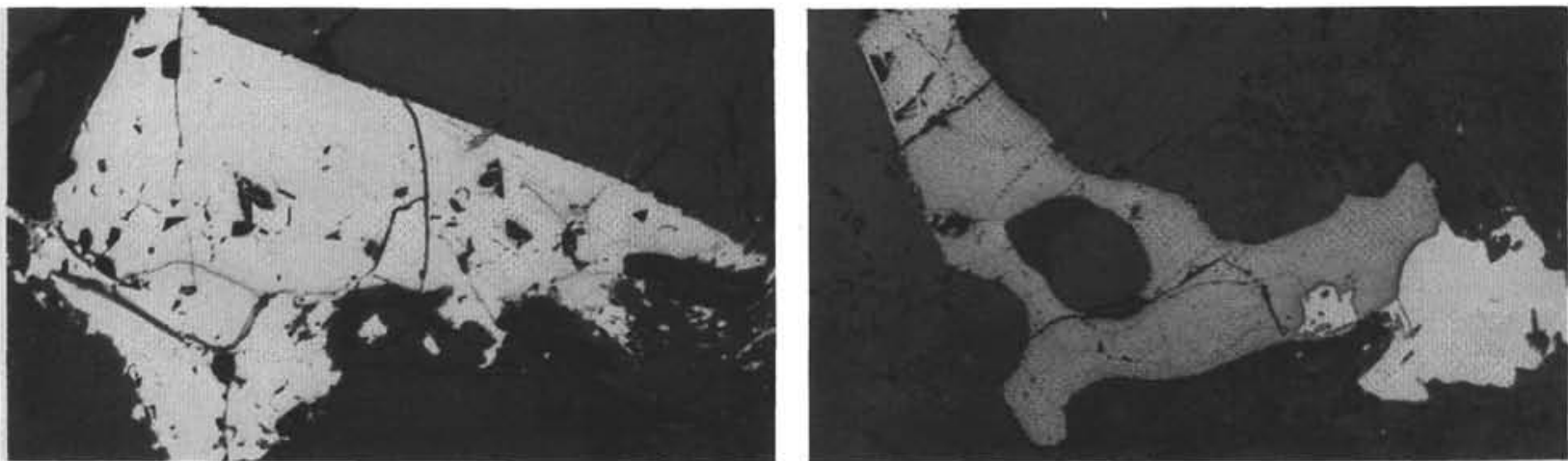

1

2
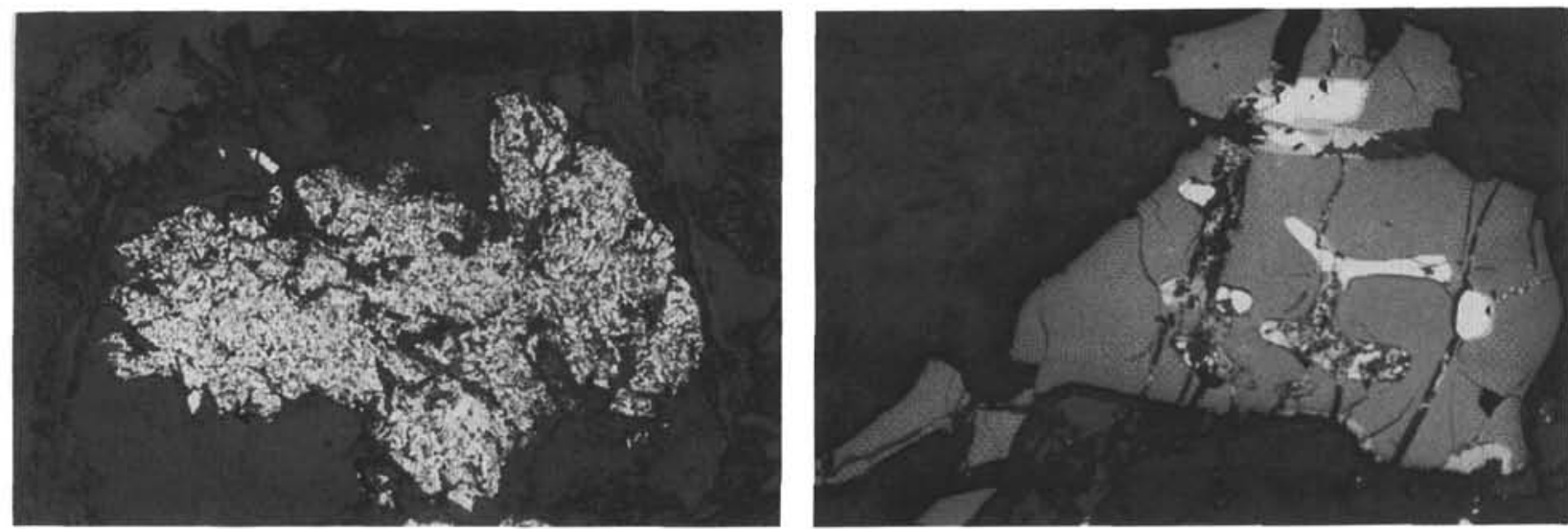

3
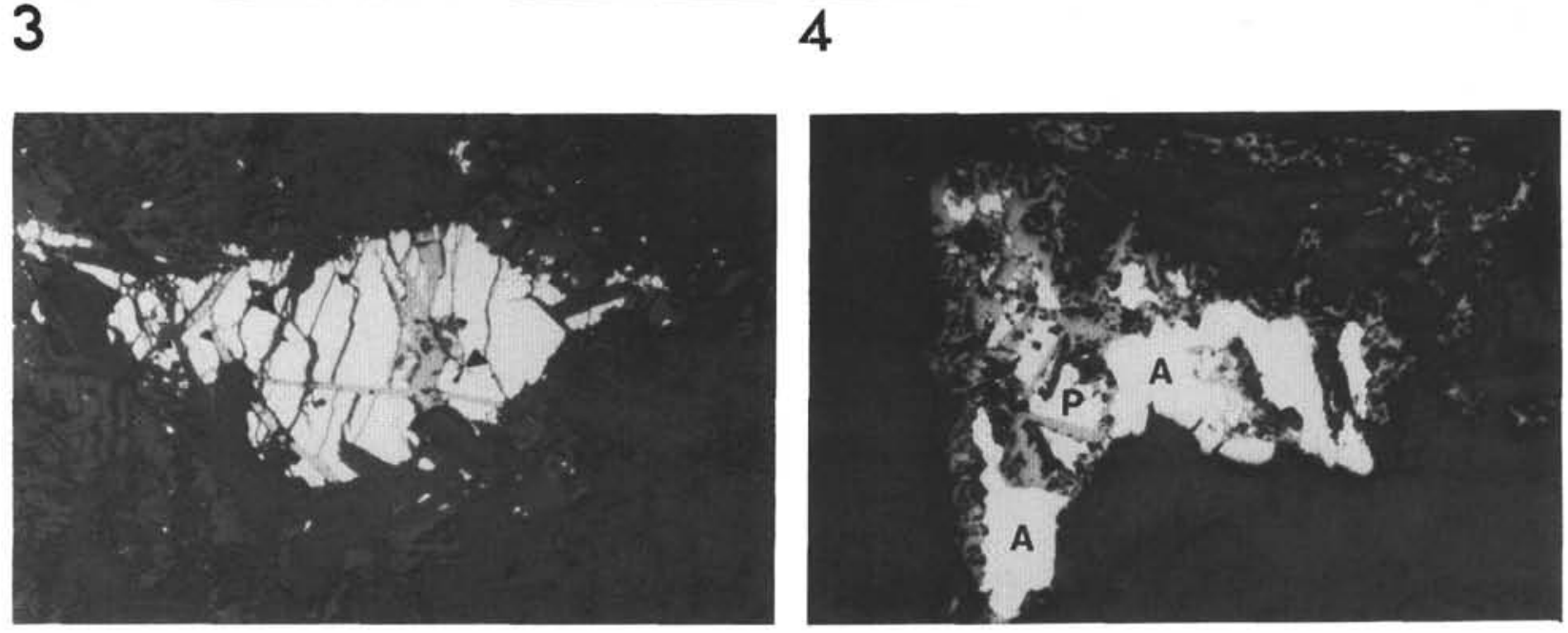

5

6

Plate 1. Photomicrographs illustrating the most common types and textures of the base-metal-bearing minerals. In all cases, the width of the field of view represents $0.5 \mathrm{~mm}$. 1. Chalcopyrite lath (analysis 1, Table 1) within an angular pyrrhotite grain (analysis 2, Table 1) from Sample 147-894G-7R-1 (Piece 11, 62-68 $\mathrm{cm})$. 2. Chalcopyrite on the edge of a rounded grain of ilmenite and magnetite interstitial to silicates from Sample 147-894G-7R-1 (Piece 11, 62-68 cm). 3. Mottled texture in an irregularly shaped grain of marcasite in an altered vein through harzburgite in Sample 147-895E-1R-3 (Piece 4B, 74-76 cm). 4. Spinel containing rounded inclusions of pentlandite. The spinel is altered in patches on its edges. From Sample 147-895D-7R-1 (Piece 11, 71-74 cm). 5. Pentlandite surrounded by serpentine and chlorite in an alteration zone between partially serpentinized olivine and totally altered plagioclase. The pentlandite shows characteristic cleavage and contains laths of magnetite. From Sample 147-895D-7R-1 (Piece 11, 71-74 cm). 6. Pentlandite (P), altering to awaruite (A), can be distinguished from awaruite by the slightly higher relief and by the presence of magnetite laths crosscutting and partially surrounding it. These base-metal minerals are close to a chrome-spinel grain, situated in a line of magnetite, and surrounded by serpentinized olivine. From Sample 147-895E-4R-2 (Piece 12, 105-109 $\mathrm{cm})$. 